\title{
ECONOMid
}

Revista Economía y Política

ISSN: 1390-7921

revista.economiaypolitica@ucuenca.edu.ec

Universidad de Cuenca

Ecuador

\section{Jóvenes universitarios y su apuesta al emprendimiento social}

Mora Pacheco, Pedro F.; Aguirre Quezada, Juan C.; Álava Atiencie, N. Gabriela; Cordero López, Juan F. Jóvenes universitarios y su apuesta al emprendimiento social

Revista Economía y Política, vol. XV, núm. 30, 2019

Universidad de Cuenca, Ecuador

DOI: https://doi.org/10.25097/rep.n30.2019.01

(c) Universidad de Cuenca 2019

Esta obra está bajo una Licencia Creative Commons Atribución-NoComercial-Compartirlgual 4.0 Internacional. 
Artículos

\section{Jóvenes universitarios y su apuesta al emprendimiento social}

Young university students and their commitment to social entrepreneurship

Pedro F. Mora Pacheco

DOI: https://doi.org/10.25097/rep.n30.2019.01

Universidad de Cuenca, Ecuador

pedro.mora@ucuenca.edu.ec

(D) http://orcid.org/0000-0002-5220-5118

Recepción: 14 Enero 2019

Juan C. Aguirre Quezada Aprobación: 08 Junio 2019

Universidad de Cuenca, Ecuador

juan.aguirreq@ucuenca.edu.ec

(D) http://orcid.org/0000-0002-8953-8151

N. Gabriela Álava Atiencie
Universidad de Cuenca, Ecuador
gabriela.alava@ucuenca.edu.ec

(iD) http://orcid.org/0002-4382-4689

Juan F. Cordero López

Universidad de Cuenca, Ecuador

juan.corderol@ucuenca.edu.ec

(iD http://orcid.org/0000-0003-0044-4333

Recepción: 14 Enero 2019

Aprobación: 08 Junio 2019

\section{Resumen:}

Este artículo tiene como propósito analizar el rol de la norma subjetiva y del ecosistema universitario, y sus efectos en la intención emprendedora de los estudiantes de las distintas carreras de la Facultad de Ciencias Económicas y Administrativas de la Universidad de Cuenca. Este trabajo de investigación es parte del proyecto internacional "Potencial para el Emprendimiento Social en América Latina" de las universidades que conforman el Accreditation Council for Business Schools and Programs (ACBSP). La investigación cuenta con una orientación metodológica cuantitativa, herramientas como la encuesta y la revisión bibliográfica y técnicas de análisis como: la regresión múltiple, la mediación estadística y estadísticos descriptivos. Los resultados indican que un ecosistema universitario adecuado motiva y refuerza la intención para llevar a cabo emprendimientos sociales, así como la aprobación por parte de familia, amigos y profesores para que el universitario emprenda, es fundamental.

Palabras clave: Ecosistema universitario, emprendimiento social, intención emprendedora, norma subjetiva, Universidad de Cuenca.

\section{ABSTRACT:}

This aims at analyzing the role of the subjective norm and the university ecosystem, and its influence on the entrepreneurial intention of students in different careers of the Faculty of Economic and Administrative Sciences at the University of Cuenca. This research work is part of the international project entitled "Potential for Social entrepreneurship in Latin America" of the universities that are part of the Accreditation Council for Business schools and Programs (ACBSP). The methodology employed in this study is quantitative and the analysis techniques include: a survey, a bibliographic review as well as multiple regression, statistical mediation, and descriptive statistics. The results indicate that an adequate university ecosystem motivates and reinforces the intention to carry out social entrepreneurships. In addition, the family, friends, and teachers approval is vital for the university student to undertake a social project.

KEYWORDS: University Ecosystem, Social Entrepreneurship, Entrepreneurial Intention, Subjective Norm, University of Cuenca. 


\section{INTRODUCCIÓN}

En los actuales escenarios de globalización es reconocido y aceptado el protagonismo que ostentan los emprendedores para alcanzar el desarrollo económico de una región o país (Toledano y Urbano, 2007; Kantis, 2010; Díaz et al, 2015; SELA, 2016).

Los emprendimientos justifican su existencia por la rapidez y flexibilidad para adaptarse a los cambios del entorno, por su potencial innovador, por su contribución a la generación de puestos de trabajo y a la diversificación del tejido productivo (Kantis, 2010; Urbano et al, 2006). Por tanto, las políticas, programas y proyectos de fomento al emprendimiento cobran vital importancia para lograr estos objetivos (OCDE, 2013; Barrado y Molina, 2015; SELA, 2016).

Teniendo como objetivo el desarrollo económico, los estados vienen realizando esfuerzos a través de política pública de incentivo al emprendimiento; es en este contexto donde las Instituciones de Educación Superior (IES) adquieren un papel protagónico por la especialización de conocimientos en torno a fomentar, preparar y motivar a sus estudiantes para emprender; esto presupone, entender a la educación como fuente de la formación de capital humano.

En los últimos años, en Latinoamérica se ha dado mucha importancia a la formación en emprendimientos a través de cursos, centros de apoyo y asesoría, incubadoras, etc. (Espíritu, 2011). Llegando, la institucionalidad educativa, a formar un círculo virtuoso junto con el estado y demás instituciones relacionadas, quienes actúan en fomento y desarrollo de la actividad emprendedora, pudiendo afirmar que la intención y las aptitudes emprendedoras llegan a forjarse a través de la educación (Formichella, 2004).

En este artículo nos proponemos analizar la capacidad explicativa de la norma subjetiva y del ecosistema universitario en la intención del emprendedor social de jóvenes estudiantes de la Facultad de Ciencias Económicas y Administrativas de la Universidad de Cuenca, como parte de la investigación sobre "Potencial para el Emprendimiento Social en América Latina" que es coordinado por la Universidad San Martín de Porres en Lima, Perú; en el marco del esquema de colaboración de las universidades que forman parte de la región 9 (Latinoamérica) del Acreditation Council for Business Schools and Porgrams (ACBSP).

La importancia de este estudio está dada por la necesidad de fortalecer la vinculación de la Universidad en un ecosistema emprendedor con una visión social que incentive a la intención emprendedora de los estudiantes. Además, con los resultados de este análisis se podrá encontrar áreas de mejora y tomar medidas que permitan optimizar el proceso de enseñanza aprendizaje.

Para el abordaje del propósito estructuramos el artículo en 5 apartados, de la siguiente manera: en el primero se realiza la introducción al tema de emprendimiento social y la necesidad de fortalecer la vinculación de la universidad como parte de un ecosistema emprendedor; en el segundo se tratan los abordajes teóricos que permiten explicar la relación entre las variables norma subjetiva, ecosistema emprendedor universitario e intención emprendedora; en el tercero se explica la metodología utilizada; en el cuarto, los resultados obtenidos de la investigación y en el quinto, se precisan elementos de discusión finales.

\section{REVISIÓN DE LITERATURA}

La pobreza y el desempleo son problemas sociales que se agravan en países que no han alcanzado el desarrollo (Samaniego, 2014). En el Ecuador, la pobreza se ha incrementado en el 2018, sobre todo en el área rural, donde llega a niveles cercanos al 40\% (RIMISP, 2018). La calidad del empleo también ha empeorado, según el Instituto Nacional de Estadísticas y Censos, INEC (2017) durante el 2016 el desempleo sube al 6,5\%; todo esto compromete a idear alternativas de solución que pueden viabilizarse desde el emprendimiento.

En este sentido, Formichella (2004) asevera que existe un círculo virtuoso entre el emprendimiento, la educación, el empleo, la pobreza y el desarrollo local: una apropiada educación otorga cualidades y competencias, lo que aumenta el espíritu emprendedor de los individuos; esto impulsa a la creación y 
desarrollo de las empresas, generando empleo y favoreciendo el desarrollo socioeconómico. El incremento de empleo permite una reducción de los recursos ociosos de la comunidad y los ciudadanos mejoran su autoestima y calidad de vida. Esta dinámica se retroalimenta por que aparecen nuevas iniciativas de proyectos (Genescá y Veciana, 1984) y se forman redes sociales que propician el desarrollo local en ese territorio (Jiao, 2011).

Por lo mencionado anteriormente, se vuelve necesario abordar el concepto de emprendedor, considerando que el protagonismo de los emprendedores en una nación pasa por el dinamismo provocado en una economía, llegando a generar oportunidades de empleo, fomento del consumo, la inversión, innovación y la competitividad, resultando a la postre en la sostenibilidad de sus organizaciones y por ende el impacto en el desarrollo local (Kantis, 2010).

Aunque, no hay un consenso en la definición del término emprendedor, las primeras definiciones de empresario surgen en 1755 (Espíritu, 2011). Garzozi et al. (2014) plantean que la palabra emprendedor se ha traducido al castellano de entrepreneur, que a su vez se origina del verbo francés entreprendre, que significa “encargarse de" (Jennings, 1994).

En el sigo XIX el autor francés Jean-Baptiste Say habla sobre la creación de valor como característica del emprendimiento (Dees, 1998). Posterior a esto, Joseph Schumpeter (1942) aporta a la definición de emprendimiento cuando incorpora el concepto de innovación como característica central de empresario relacionado con el crecimiento económico. Partiendo de los postulados de Say y Schumpeter, es Peter Drucker (1982) quien ofrece un nuevo enfoque definiendo a los emprendedores como explotadores de oportunidades.

En la actualidad se reconoce en el emprendedor la capacidad de comenzar y operar nuevas empresas (Hurtado, 2016) y también, la capacidad de generar éxito con su accionar, derivando sus iniciativas en rentabilidad (Lederman et al, 2014; Alcaraz, 2011). Es así que, el emprendimiento es una mezcla de oportunidad, creatividad, innovación y liderazgo para crear valor a partir de una idea. Dicha definición, como han sugerido algunos autores (Drucker, 1985; López de Toro, 2014), genera una convergencia en los distintos tipos de emprendimiento, sean de carácter económico como de carácter social.

Desde un enfoque multidimensional, no todo puede resumirse al emprendimiento "tradicional", con fines de lucro, en donde prima el individualismo obtenido en los resultados empresariales, sino existirán factores de mucha más representatividad colectiva como el bienestar, la satisfacción de necesidades hacia la sociedad y el aporte a los grandes grupos vulnerables; de ahí que, el emprendimiento es concebido como la fuente de varias aristas según la finalidad que persigan siendo protagonista el emprendimiento social cuando se habla de resolver conflictos y ayudar a los sectores sociales (Rodríguez y Larrota, 2013).

Los emprendimientos sociales son concebidos como iniciativas empresariales que utilizan toda la gama de herramientas sociales para su operación, comandadas por los principios de eficiencia, eficacia y economía empresarial, maximización de la riqueza para alcanzar un objetivo social (Vernis \& Iglesias, 2010). Las definiciones de emprendimiento social han sido generadas de varias formas, partiendo desde su misión social y su sostenibilidad como eje vertebral de este concepto, llegando a definirlo como un catalizador de la transformación social (Alvord, Brown \& Letts, 2004), o como lo define Zahra et al. (2009) el: “...descubrir, definir y aprovechar las oportunidades para mejorar la riqueza social...” (p.519).

Para López de Toro (2014) el emprendimiento clásico y el emprendimiento social comparten la búsqueda y explotación de oportunidades desde la innovación, para provocar cambios que permitan alcanzar sus objetivos; tienen elementos comunes como la asunción de riesgos, la creatividad, la persistencia y la voluntad, permitiéndoles ser agentes de cambio. Sin embargo, los emprendedores sociales tienen una misión específica que es crear y sostener valor social. Lo más importante de su emprendimiento es su misión social, que se concreta en acciones que resuelvan necesidades y retos sociales.

Por tanto, el emprendimiento social se concibe desde un enfoque multidimensional de apoyo al colectivo, sostenibilidad y de estructura organizacional (como medio), hecho que actúa con variabilidad de perspectivas 
de acuerdo al énfasis realizado sobre sus dimensiones, llegando a establecerse una prioridad de entre sus factores (Guzmán y Trujillo 2008:110).

Múltiples estudios han capturado la relación empírica positiva y significativa existente entre programas de apoyo universitarios en pro del emprendimiento y la intención de emprender de los jóvenes en aquellos centros; Bechard, y Toulouse (1988), manifiestan expresamente el estímulo hacia la intención emprendedora venida desde la formación formal. Según Brito, Cruz y Hernández (2014) definen a la intención desde su utilidad, calificándola como "el mejor elemento para predecir el comportamiento planeado".

El concepto aplicado a la empresarialidad recoge un sin número de aportes, sin embargo, es conducente a la creación de modelos de intención que prevengan sobre el comportamiento tomado por los emprendedores frente a su contexto; los más utilizados son los creados por Shapero \& Sokol (1982) y Ajzen (1991), tratando de perfilar estos hacia la predicción de la intención emprendedora. Se la define también, como la actitud presentada ante el emprendimiento por las personas que muestran interés en aquello; de forma más específica y técnica, Prodan y Drnovsek (2010) la definen como el estado mental en el que el individuo centra su interés en el objetivo de la creación de una empresa.

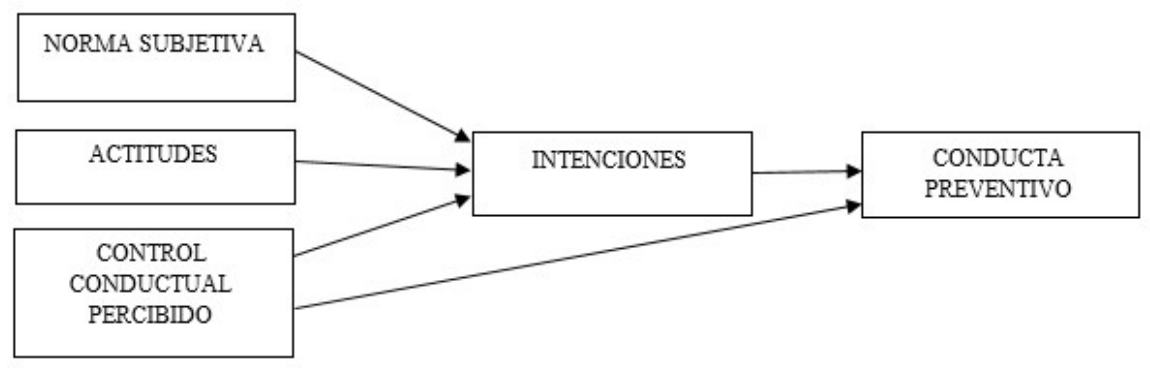

\section{GRÁFICO 1. \\ Modelo de conducta planificada \\ Ajzen (1991).}

Como se observa en el gráfico 1, la Teoría de Acción Planificada o TPB de Ajzen (1991) plantea que los elementos que influyen en la conducta son: la norma subjetiva o el grado en que una persona está motivada a cumplir con los paradigmas de la gente que le rodea; la actitud hacia la conducta y, los controles internos y externos percibidos. Estos elementos generan las intenciones que a su vez llevan a la conducta preventiva.

En la búsqueda de este conocimiento se incluyó en algunas investigaciones a los estudiantes como objeto de análisis fundamental por sus características de comportamiento y su contexto (Harrison \& List, 2004; Sáez, Jiménez y Díaz, 2015). La educación generalmente se considera como un factor incidente directamente sobre la intención de emprender (Toledano, 2006), que podría alimentarse en los jóvenes universitarios (Liseras, 2003), pues se contará con el análisis directo también del capital humano especializado, tan necesario para la mejora empresarial. Cuando hablamos del entorno universitario se encuentran jóvenes, que por sus características, son mucho más accesibles al cambio, definiéndose favorabilidad para el emprendimiento (García et al., 2006), por lo que, el clima será trascendental al momento de estudiar la intención emprendedora (Díaz-Bretones et al., 2011); en este sentido las universidades podrían ser los centros más aptos para mejorar dicha intencionalidad.

Pizarro (2003) señala que la educación potencializa la capacidad de reorganizarse y tomar decisiones que causen beneficio próximo, por lo que se considera a la educación compleja pero además participativa (Echeverría, 2009), involucrando a todos los actores de forma tal que les ocasione un mejoramiento de su situación actual, ocupando las diferentes dimensiones de la educación (Torres, 2001; Díaz et al., 2015; SoriaBarreto, 2016)y llegando a establecerse como una variable de amplio análisis frente a la favorabilidad de presentar un clima universitario comprometido con el emprendimiento; asumiendo para los estudiantes mayores conocimientos y habilidad canalizados a través de la práctica (Duval-Couetil, 2013); generando 
la necesidad de la flexibilidad del aprendizaje universitario forjado desde las especificidades mismas de los estudiantes.

Por su parte, la norma subjetiva mide la presión social percibida para llevar a cabo o no la conducta (Ajzen, 1991). Aplicado al emprendimiento, estas normas se refieren a la percepción del grado de aprobación de la decisión de emprender de las personas de referencia del sujeto analizado (De Luco, 2014). Para el estudiante universitario estas normas miden la presión de su entorno inmediato a la hora de optar o no por el emprendimiento, por la valoración que le otorga su familia, amigos y colegas a la aventura de emprender (Brito, et al., 2014).

Liñán y Chen (2009) establecen la importancia de la norma subjetiva, es decir, ese tipo de incentivo o impacto que recibe el estudiante en cuanto a la opinión en su contexto sobre emprendimientos, demostrándose la determinación de la relación positiva de esta, con la intención emprendedora, en donde coexisten factores determinantes que incentivan o no la toma de decisiones generada por los emprendedores universitarios (Ajzen, 2002), llegando a definirse, en contexto la importancia trascendental que tiene el entorno y su opinión en los emprendimientos.

Ese entorno puede configurar un ecosistema emprendedor; forjado como uno de los principales objetivos de política en la actualidad, apuntalándose en la sostenibilidad del emprendimiento a través de individuos formados, visionarios, instituciones, condiciones y demás factores que a la postre determinarán el fomento del emprendimiento.

El ecosistema emprendedor es definido como el entorno que rodea a las organizaciones o a los individuos que se encuentran inmersos en el campo del emprendimiento e impacta en su intención emprendedora (Liñán, 2006), razón por la cual las universidades juegan un papel primordial en este entorno, pues son capaces de fomentar, preparar y motivar a sus estudiantes para emprender (Espíritu, 2011).

El análisis del ecosistema de emprendimiento en las instituciones de educación superior (IES), se centra en la intención emprendedora de los estudiantes, el perfil del docente, las estrategias de fomento y los planes de negocio para establecer la línea base del desarrollo de la cultura del emprendimiento. La universidad potencia el capital humano de sus estudiantes en dos niveles: la difusión de la cultura emprendedora y el desarrollo de competencias específicas para emprender (Caicedo, et al. 2015), sin duda, el fomento del emprendimiento dependerá de un sin número de factores en una nación, en este contexto, el clima o ecosistema emprendedor podría ser decisor al momento de construir un tejido emprendedor sostenible.

Diferentes teorías e investigaciones construidas hace varios años demuestran la relación e influencia directa que tendrá el entorno con la conducta de un individuo, y hablan sobre la importancia y significatividad de la intención emprendedora al momento de analizar un emprendimiento, considerando para ello el accionar de la universidad en este contexto (Liñán \& Chen, 2009; Veciana, \& Urbano, 2004; Aponte, Urbano y Veciana, 2006; Díaz et al., 2015; Cervantes, et al., 2016; Espíritu, 2011).

Para comparar experiencias, teorías y opiniones, con puntos de vista diversos y complementarios a esta investigación, se reseñan algunas investigaciones ya realizadas anteriormente:

- Astudillo, Mora y Pozo (2019) realizan una evaluación de la cátedra de emprendimiento en varias carreras del área social de la Universidad de Cuenca, Ecuador, con test aplicados al inicio y al final del ciclo lectivo. Los resultados permiten afirmar que los estudiantes, al finalizar la cátedra, perciben un incremento de sus conocimientos, habilidades e información de su potencial emprendedor en comparación con el inicio, sin embargo, esta diferencia no es significativa en el caso de las habilidades y conocimientos, aunque si lo es respecto a la información sobre los programas, procedimientos y mecanismos de ayuda.

- Giraldo-Mejía y Vara-Horna (2018) coordinan una investigación que abarca 26 universidades de 8 países de Latinoamerica: Argentina, Chile, Colombia, Ecuador, México, Paraguay, Perú y República Dominicana; en la misma se plantea la interrogante ¿Están las universidades haciendo lo suficiente sobre el emprendimiento social? Ellos argumentan que, aunque hay avances en la formación universitaria en Emprendimiento social, aún no es suficiente. Identifican dos datos relevantes; el primero es que a pesar de 
que entre el $62 \%$ y el $66 \%$ de los estudiantes tiene la intención y ha recibido los insumos necesarios para emprender, solamente el 3\% está desarrollando un emprendimiento social.

- Bilbao y Vélez (2015) analizan las competencias necesarias para que los estudiantes universitarios puedan realizar un emprendimiento social; para ello, contraponen la teoría con los resultados de la investigación Alfa-Tuning Latin American Project 2011-2013. En esta investigación se revisan siete programas de emprendimiento social de varias universidades de España y de América Latina: Universidad Católica de Temuco en Chile, Universidad de Deusto en Bilbao, España, Pontificia Universidad Javeriana de Cali, Colombia, Universidad de Paraná, en Brasil. Las competencias que se necesitan se agrupan en cuatro bloques: competencias relacionadas con la tarea o trabajo, competencias de relación con los demás, competencias personales y competencias relacionadas con la faceta filosófica y ética.

- Astudillo et al. (2019) citan otras investigaciones sobre la intención emprendedora en centros universitarios como la de Cervantes et al (2016) en México y la de Díaz et al (2015) en España, donde la formación académica incrementa la motivación para emprender.

- Espíritu (2011) investiga sobre la importancia que tienen los rasgos psicológicos de personalidad y características sociodemográficas en la intención emprendedora de estudiantes de una universidad de Colima en México. Los resultados encontrados son que tres rasgos de personalidad: necesidad de logro, locus de control interno y propensión al riesgo muestran una alta relación positiva con respecto a la intención de crear su propia empresa.

- Toledano (2006) realiza una investigación sobre las actitudes hacia la creación de empresas de estudiantes de la Universidad de Huelva, España; se concluye en esta investigación que el proceso de formación de emprendedores aun es incipiente. Se considera que este resultado se presenta por la cultura de esa región que a criterio de los investigadores prefieren la actividad asalariada o la función pública. Es interesante, en este trabajo; la recomendación que le hacen a la universidad en el sentido de que debería profundizar institucionalmente programas de fomento del espíritu emprendedor no solo a los estudiantes sino también a los docentes.

- Tres investigaciones: Genescá y Veciana (1984), Veciana y Urbano (2004) y Aponte, Urbano y Veciana (2006); se centraron en las actitudes de estudiantes universitarios para crear empresas y compararon los resultados obtenidos en Catalunya (España) y Puerto Rico (Estados Unidos); los resultados encontrados no presentan mayores diferencias entre los estudiantes de Europa o de Norteamérica; en ambas regiones, los universitarios presentan motivación emprendedora, aunque son pesimistas respecto a su viabilidad.

Tomando en cuenta las consideraciones de este ítem, para esta investigación, se plantean las siguientes hipótesis:

H1.- La norma subjetiva es determinante para que se genere una intención hacia un emprendimiento social

H2.- El ecosistema es un factor que potencia la intención emprendedora

\section{Materiales Y MÉTODOS}

En el presente trabajo el enfoque metodológico utilizado fue el cuantitativo y se utilizaron 3 variables de constructo debidamente validadas que corresponden a norma subjetiva, ecosistema emprendedor universitario e intención emprendedora. La variable dependiente que se explica es la intención emprendedora en base a las variables: norma subjetiva y el ecosistema emprendedor universitario que se corresponden a las variables independientes. 


\subsection{Unidad de análisis}

La unidad observada de la que se levantó la información primaria corresponde a los y las jóvenes universitarios(as) de la Facultad de Ciencias Económicas y Administrativas de la Universidad de Cuenca en el ciclo Septiembre 2017 - Febrero 2018 que cursaban los últimos ciclos de cada una de las seis carreras de la Facultad; esto es desde el sexto hasta el noveno ciclo.

\subsection{Población objeto de estudio}

La población objeto de estudio la constituyen un total de 407 estudiantes matriculados en los últimos ciclos de las seis carreras de la Facultad de Ciencias Económicas y Administrativas,el número de estudiantes por ciclo que cursan las diferentes carreras, en el periodo de estudio, son (tabla 1):

TABLA 1.

Población de estudiantes matriculados de sexto a noveno ciclo

\begin{tabular}{|l|l|}
\hline Carreras & $\begin{array}{l}\text { Nro. de } \\
\text { Estudiantes }\end{array}$ \\
\hline $\begin{array}{l}\text { Administración de } \\
\text { Empresas }\end{array}$ & 107 \\
\hline $\begin{array}{l}\text { Contabilidad y } \\
\text { Auditoria }\end{array}$ & 163 \\
\hline Economía & 29 \\
\hline $\begin{array}{l}\text { Ingeniería } \\
\text { Empresarial }\end{array}$ & 36 \\
\hline Marketing & 18 \\
\hline Sociología & 54 \\
\hline TOTAL & 407 \\
\hline
\end{tabular}

Registros Facultad de Ciencias Económicas y Administrativas Universidad de Cuenca período Septiembre/2017 - Febrero/2018

\subsection{Instrumento utilizado}

La herramienta empleada en el presente trabajo fue el cuestionario para medir el perfil emprendedor social utilizado en el marco del proyecto "Potencial para el Emprendimiento Social en América Latina” promovido por el Instituto de Investigación de la Facultad de Ciencias Administrativas de la Universidad San Martín de Porres (USMP, 2017) Lima-Perú; herramienta que analiza una gama de conductas emprendedoras sociales como son: la percepción emprendedora, orientación emprendedora, intención emprendedora, autoeficacia, norma subjetiva y ecosistema emprendedor universitario.

Para nuestro análisis, se tomaron las escalas de las variables:

Intención emprendedora social.- Es una escala unidimensional conformada por 5 ítems en escala Likert que tiene como objetivo medir la práctica de jóvenes universitarios en generar emprendimientos desde una perspectiva social. La escala con la que se trabaja se basa en Moriano (2005) y Liñán y Chen (2009) citado por Giraldo W. \& Vara-Horna A. (2018, p. 60).

Norma subjetiva del emprendedor social.- Es una escala multidimensional con 3 sub-dimensiones que corresponden a las variables familia, amigos y profesores que tiene como objetivo analizar la aprobación social por parte de familiares, amigos y profesores en llevar a cabo emprendimientos desde una perspectiva social. La escala para medir el constructo es de 7 ítems en escala Likert basados en Moriano (2005) y Sánchez-Almagro (2003) citado por Giraldo W. \& Vara-Horna A. (2018, p. 60). 
Ecosistema emprendedor universitario.- Es una escala unidimensional de 9 ítems en escala Likert que tiene como objetivo analizar las condiciones del ecosistema emprendedor universitario para generar habilidades emprendedoras desde una perspectiva social. La escala utilizada se basa en Lüthje \& Franke (2004), traducidas al español por Álvarez, López y Chafloque (2018), citado por Giraldo W. \& Vara-Horna A. (2018, p. 60).

Con respecto a la fiabilidad y validez de las escalas utilizadas para analizar las variables de constructo: intención emprendedora, norma subjetiva y ecosistema emprendedor universitario, en la Tabla 2 se presentan los datos provenientes de la información receptada a jóvenes universitarios de la Facultad de Ciencias Económicas y Administrativas de la Universidad de Cuenca que demuestran altos niveles de fiabilidad y validez. La consistencia interna medida a través del Alpha de Cronbach está entre 0.856 a 0.925 , la consistencia o fiabilidad compuesta de los constructos está entre 0.863 a 0.938 ; con respecto a la validez convergente, las cargas factoriales son superiores a 0.461 y las varianzas explicadas están por encima de 0.48 , medidas que verifican la fiabilidad y validez de los constructos empleados para el presente trabajo.

TABLA 2.

Fiabilidad y validez de las escalas de medición intención emprendedora, norma subjetiva y ecosistema emprendedor universitario

\begin{tabular}{|c|c|c|c|c|c|}
\hline Nro. & Sub Escalas & $\begin{array}{l}\text { Alpha } \\
\text { Cronbach }\end{array}$ & $\begin{array}{l}\text { Fiabilidad } \\
\text { Compuesta } \\
\text { (FC) }\end{array}$ & $\begin{array}{l}\text { Cargas } \\
\text { factoriales } \\
\text { (rango) }\end{array}$ & $\begin{array}{l}\text { Varianza } \\
\text { extraida } \\
\text { media } \\
\text { AVE }\end{array}$ \\
\hline 1 & $\begin{array}{l}\text { Intención } \\
\text { Emprendedora }\end{array}$ & 0,856 & 0,897 & $\begin{array}{l}0,737- \\
0,863\end{array}$ & 0,635839 \\
\hline \multirow[t]{4}{*}{2} & $\begin{array}{l}\text { Norma } \\
\text { Subjetiva } \\
\text { (segundo } \\
\text { orden) }\end{array}$ & --- & 0,863 & $\begin{array}{l}0,461- \\
0,779\end{array}$ & 0,478518 \\
\hline & Familia & 0,666 & 0,857 & 0,866 & 0,750098 \\
\hline & Amigos & 0,437 & 0,781 & 0,801 & 0,641351 \\
\hline & Profesores & 0,799 & 0,882 & $\begin{array}{l}0,827- \\
0.878\end{array}$ & 0,713969 \\
\hline 3 & $\begin{array}{l}\text { Ecosistema } \\
\text { Emprendedor } \\
\text { Universitario }\end{array}$ & 0,925 & 0,938 & $\begin{array}{l}0,673- \\
0,870\end{array}$ & 0,629763 \\
\hline
\end{tabular}

Base de datos en SPSS versión 20 del Cuestionario de Emprendimiento Social en Latinoamérica aplicado a jóvenes universitarios de la Facultad de Ciencias Económicas y Administrativas de la Universidad de Cuenca - Ecuador

\subsection{Técnicas de análisis}

Para el análisis de datos se utilizaron los programas informáticos: Statistical Package for the Social Sciences (SPSS) utilizado en gran medida en investigaciones con enfoque cuantitativo en las ciencias sociales y aplicadas, y en el ámbito empresarial. Así también, se utiliza el Partial Least Square software Smart PLS 3, que se considera una herramienta potente para el análisis de datos multivariados (Ayaviri, Melo y Saucedo, 2018), sin basarse en la covarianza (LISREL o AMOS) sino más bien por estimación de mínimos cuadrados ordinarios y componentes principales. La investigación en administración, emprendimiento, empresas, etc., cada vez se ha sofisticado y ha incluido herramientas más precisas y flexibles para el análisis de datos, es así que, los modelos de ecuaciones estructurales cobran protagonismo entre las investigaciones de índole empresarial al ser una extensión de los modelos multivariantes (Chin, 1998) y sobre todo la técnica PLS por las ventajas que presenta respecto a tamaño de la muestra, tipo de variables y su distribución, además de la maximización de la varianza explicada $\left(\mathrm{R}^{2}\right)$ y su poco requerimiento respecto a las distribuciones residuales (Cepeda y Roldán, 2004) 
El análisis basado en PLS resulta adaptarse de una manera óptima al desarrollo de la teoría (análisis exploratorio) y además al realizar análisis predictivos (Barclay et al., 1995; Chin et al., 2003).

Cepeda y Roldán (2004) citan a revistas indexadas vinculadas a Administración de Empresas que han recogido investigaciones relacionadas al uso de la técnica, por lo que se procede a incluir un listado con algunas de aquellas:

- Accounting, Organizations \& Society

- Administrative Science Quarterly

- International Journal of Researchin Marketing

- Journal oflnternational Financial Management \& Accounting

- Management Science

- Total Quality Management \& Business Excellence

De esta manera, las técnicas utilizadas para el procesamiento de información mediante los programas informáticos, antes mencionados fueron:

Estadísticos descriptivos.- Se obtuvieron proporciones muestrales en base a las variables sexo y actividad actual con la finalidad de describir la situación de los jóvenes universitarios en estas dos variables.

Regresión múltiple.- Se utilizó la variable de constructo intención emprendedora como variable dependiente a explicar en base a las variables independientes de constructo norma subjetiva y ecosistema emprendedor universitario.

Mediación estadística.- Se identificó la influencia indirecta o mediación que ejerce la variable independiente ecosistema emprendedor universitario en la variable dependiente intención emprendedora.

\section{Resultados}

En la Tabla 3, se observa que la mayoría de universitarios únicamente se dedican a estudiar en un porcentaje del 72,7\% que corresponde a 176 estudiantes de los 242 investigados, en comparación con los que estudian y trabajan. Otro aspecto que se destaca, es que en su mayoría son de sexo mujer en un porcentaje del $61,2 \%$ y al analizar la probabilidad conjunta se destacan las variables que estudie y sea de sexo mujer en un porcentaje del 46,7\% que corresponden a 113 estudiantes. (Error 1: La referencia debe estar ligada) (Error 2: El tipo de referencia es un elemento obligatorio) (Error 3: No existe una url relacionada)

TABLA 3.

\section{Estadísticos descriptivos por sexo y actividad actual}

\begin{tabular}{|l|l|l|l|l|l|l|}
\hline Sexo & \multicolumn{9}{l}{ Actividad Actual } \\
\cline { 2 - 7 } & Estudia & $\begin{array}{l}\text { Estudia y } \\
\text { trabaja }\end{array}$ & \multicolumn{2}{l|}{ Total } \\
& Nro. & $\%$ & Nro. & $\%$ & Nro. & $\%$ \\
\hline Hombre & 63 & $26,0 \%$ & 31 & $12,8 \%$ & 94 & $38,8 \%$ \\
\hline Mujer & 113 & $46,7 \%$ & 35 & $14,5 \%$ & 148 & $61,2 \%$ \\
\hline Total & 176 & $72,7 \%$ & 66 & $27,3 \%$ & 242 & $100 \%$ \\
\hline
\end{tabular}

\footnotetext{
Base de datos en SPSS versión 20 del Cuestionario de Emprendimiento Social en Latinoamérica aplicado a jóvenes universitarios de la Facultad de Ciencias Económicas y Administrativas Universidad de Cuenca-Ecuador.
}

En la Tabla 4 se analiza la capacidad explicativa de las variables independientes norma subjetiva y ecosistema emprendedor universitario en la variable dependiente intención emprendedora, identificándose que ambas variables de forma significativa explican la intención emprendedora social con valor $p$ de 0,000 y 0,010 respectivamente. En el caso de la norma subjetiva se observa que la capacidad explicativa es extremadamente fuerte en la intención emprendedora; mientras que el ecosistema emprendedor 
universitario explica que la intención emprendedora es de forma fuerte. Ambas variables independientes proporcionan una varianza explicada del $19.8 \%$ y el $80.2 \%$ se debe a otros factores. Dicho de otra manera, tanto la motivación para obtener aprobación de las personas del entorno, como las estrategias metodológicas, perfil docente y los planes de negocio de la universidad, inciden en la intención emprendedora del joven universitario; sin embargo, la presión percibida de las personas de referencia del joven pesa más que el plan de estudios universitario.

TABLA 4.

Capacidad explicativa de la norma subjetiva y del ecosistema emprendedor universitario en la intención emprendedora.

\begin{tabular}{|c|c|c|c|}
\hline & \multicolumn{3}{|c|}{$\begin{array}{l}\text { Intención emprendedora (variable } \\
\text { dependiente) }\end{array}$} \\
\hline & $\begin{array}{l}\text { Coeficiente } \\
\text { Correlación } \\
\mathrm{R}\end{array}$ & Valor $p$ & $\begin{array}{l}\text { Coeficiente } \\
\text { Determinación } \\
\text { R2 }\end{array}$ \\
\hline $\begin{array}{l}\text { Norma subjetiva } \\
\text { (aprobación social) } \\
\text { (variable } \\
\text { independiente) }\end{array}$ & 0,453 & $0,000^{* *}$ & \multirow{2}{*}{0,198} \\
\hline $\begin{array}{l}\text { Ecosistema } \\
\text { emprendedor } \\
\text { universitario (variable } \\
\text { independiente) }\end{array}$ & 0,165 & $0,010^{*}$ & \\
\hline
\end{tabular}

\footnotetext{
Base de datos en SPSS versión 20 del Cuestionario de Emprendimiento Social en Latinoamérica aplicado a jóvenes universitarios de la Facultad de Ciencias Económicas y Administrativas de la Universidad de Cuenca - Ecuador

${ }^{* *}$ El coeficiente de correlación y regresión es significativo al nivel 0,01 (bilateral).

* El coeficiente de correlación y regresión es significativo al nivel 0,05 (bilateral).
}

Por otra parte, en la Tabla 5 se desagrega las sub-dimensiones de segundo orden de la norma subjetiva, que con la variable unidimensional ecosistema emprendedor universitario analizan la capacidad explicativa; a través de la técnica de regresión múltiple. Observándose que las sub-dimensiones de norma subjetiva familia y profesores son las que explican de forma significativa la intención emprendedora con un valor $p$ de 0,000 y 0,019 respectivamente. En la que la aprobación de la familia muestra una explicación extremadamente significativa en la intención emprendedora y la aprobación social de profesores explican de forma fuerte la intención emprendedora. Considerando cada una de las 3 sub-dimensiones de norma subjetiva: familia, amigos y profesores; así como, ecosistema emprendedor universitario variable unidimensional como variables independientes que proporcionan una varianza explicada del 21,8\% al constructo intención emprendedora. 
TABLA 5.

Capacidad explicativa de las sub-dimensiones de norma subjetiva en la intención emprendedora de jóvenes universitarios

\begin{tabular}{|l|l|l|l|l|}
\hline \multicolumn{3}{|c|}{} & \multicolumn{3}{|l|}{$\begin{array}{l}\text { Intención emprendedora (Variable } \\
\text { dependiente) }\end{array}$} & $\begin{array}{l}\text { Beta } \\
\text { estandarizado }\end{array}$ & Valor p & $\begin{array}{l}\text { Coeficiente } \\
\text { Determinación } \\
\text { R2 }\end{array}$ \\
\hline Dimensión & Sub-dimensiones & & \\
\hline $\begin{array}{l}\text { Norma } \\
\text { Subjetiva } \\
\text { (aprobación } \\
\text { Social) } \\
\text { Variable } \\
\text { independiente) }\end{array}$ & Familia & 0,377 & $0,000^{* *}$ & 0,218 \\
\cline { 2 - 4 } & Amigos & $-0,006$ & 0,928 & \\
\hline $\begin{array}{l}\text { Ecosistema } \\
\text { emprendedor } \\
\text { universitario } \\
\text { Variable } \\
\text { independiente) }\end{array}$ & Profesores & 0.166 & $0,019^{*}$ & \\
\hline
\end{tabular}

\footnotetext{
Base de datos en SPSS versión 20 del Cuestionario de Emprendimiento Social en Latinoamérica aplicado a jóvenes universitarios de la Facultad de Ciencias Económicas y Administrativas de la Universidad de Cuenca - Ecuador.

${ }^{* *}$ El coeficiente de correlación y regresión es significativo al nivel 0,01 (bilateral).

* El coeficiente de correlación y regresión es significativo al nivel 0,05 (bilateral).
}

Sin embargo, en la Tabla 6 observamos que al eliminar la variable unidimensional ecosistema emprendedor universitario del análisis y trabajar únicamente con las 3 sub-dimensiones de norma subjetiva: familia, amigos y profesores como variables independientes la varianza explicada en intención emprendedora mejora a una capacidad explicativa del 22,1\%; mientras que los valores $p$ se presentan en 0,$000 ; 0,000 ; 0,000$ respectivamente en cada una de las 3 variables explicando de forma extremadamente significativa la variable de constructo intención emprendedora. En otras palabras, en ausencia del ecosistema de emprendimiento universitario, la aprobación social de las personas referentes del individuo, familia, amigos y profesores, juegan un rol fundamental en la explicación de su intención emprendedora.

TABLA 6.

Capacidad explicativa de las sub-dimensiones de norma subjetiva en la intención emprendedora de jóvenes universitarios

\begin{tabular}{|l|l|l|l|l|}
\hline \multicolumn{3}{|c|}{} & \multicolumn{2}{l|}{$\begin{array}{l}\text { Intención emprendedora (Variable } \\
\text { dependiente) }\end{array}$} \\
\cline { 3 - 4 } & $\begin{array}{l}\text { Beta } \\
\text { estandarizado }\end{array}$ & Valor $p$ & $\begin{array}{l}\text { Coeficiente } \\
\text { Determinación } \\
R^{2}\end{array}$ \\
\hline Dimensión & Sub-dimensiones & & & \\
\hline $\begin{array}{l}\text { Norma subjetiva } \\
\text { (aprobación social) } \\
\text { (Variable independiente) }\end{array}$ & Familia & 0,377 & $0,000^{* *}$ & 0,221 \\
\cline { 2 - 4 } & Amigos & $-0,004$ & $0,000^{* *}$ & \\
\hline
\end{tabular}

Base de datos en SPSS versión 20 del Cuestionario de Emprendimiento Social en Latinoamérica aplicado a jóvenes universitarios de la Facultad de Ciencias Económicas y Administrativas de la Universidad de Cuenca - Ecuador. ${ }^{* *}$ El coeficiente de correlación y regresión es significativo al nivel 0,01 (bilateral).

Como se observa en el gráfico 2, al incorporar la variable de constructo ecosistema emprendedor universitario como mediadora entre la variable independiente norma subjetiva y la variable dependiente 
intención emprendedora se verifica la influencia indirecta que tiene esta variable mediadora para mejorar la relación entre las variables descritas, reflejado en el 16,7\% de varianza explicada. Razón por la que el ecosistema de emprendimiento universitario es significativo cuando se aporta sobre la intención de realizar emprendimientos con un abordaje social. El modelo testeado reporta de forma conjunta una varianza explicada de $22.1 \%$

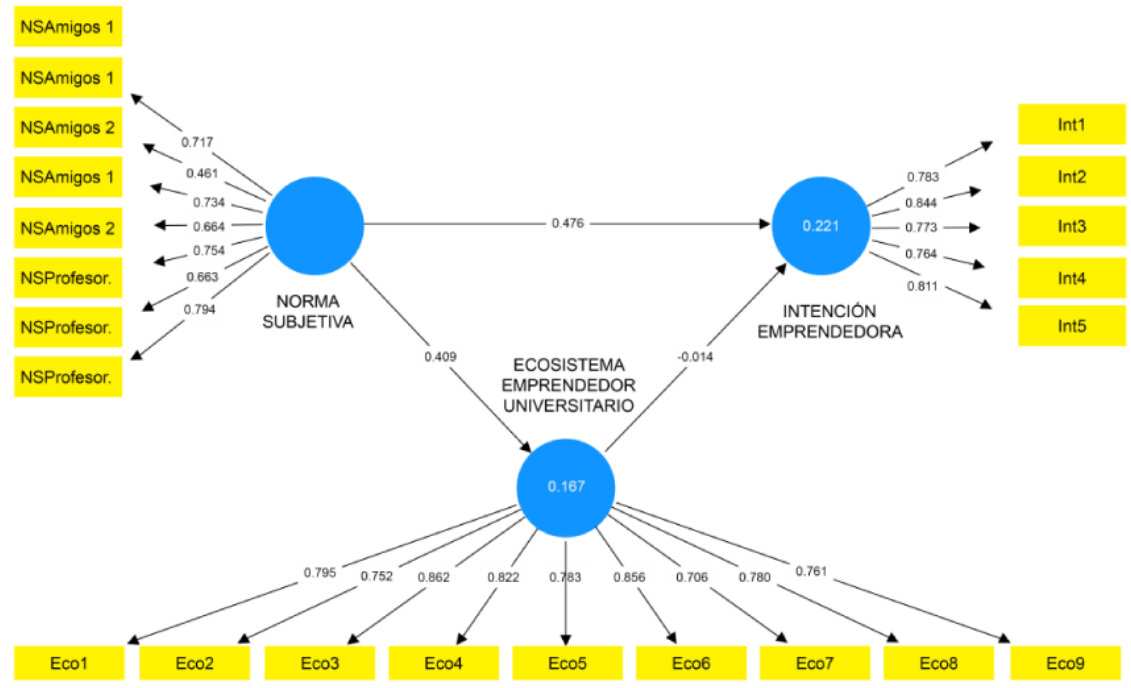

GRÁFICO 2.

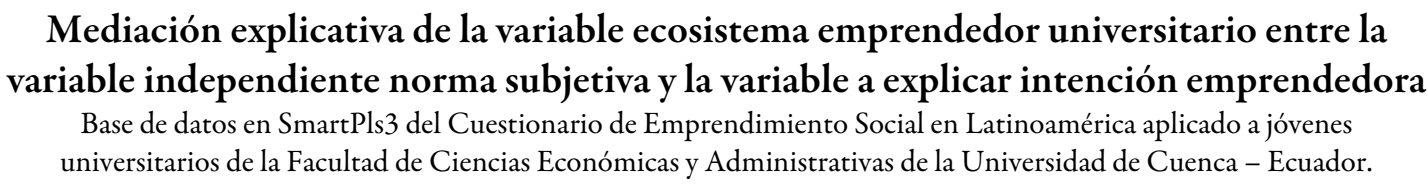

\section{ConClusiones Y RECOMENDACIONES}

En la presente investigación se explica la intención emprendedora social a partir de la norma subjetiva y el ecosistema emprendedor universitario; en este marco, se determinó analizar diversas características personales y de contexto, la formación recibida y el marco institucional universitario con la actitud emprendedora de los estudiantes universitarios.

En la revisión bibliográfica, se encontraron diversas investigaciones sobre la intención emprendedora de estudiantes universitarios, el incremento de estos estudios evidencia la importancia que se le está dando en los círculos académicos a este tema y la necesidad de investigaciones más exhaustivas. Una de las características relevantes de este trabajo es la forma que se han hecho operativos los ítems, variables y las dimensiones analizadas, ya que al haber elegido la bibliografía se hizo necesario justificar cada una de ellos y hacer operativa la capacidad interpretativa de las teorías que explican la actitud emprendedora.

Al construir las variables se utilizaron los ítems del proyecto de investigación colaborativo "Potencial para el Emprendimiento Social en América Latina" del Accreditation Council for Business Schools and Programs (ACBSP).

Los resultados indican que la intención emprendedora se ve influenciada significativamente, en nuestro contexto, por la variable norma subjetiva y sus tres sub-dimensiones, sobre todo por la familia y los profesores; constructos que fueron verificados en su fiabilidad y validez. Estos resultados guardan sintonía con otros estudios e investigaciones realizados por Ajzen (1991), Pizarro (2003), Díaz et al. (2015), Soria - Barreto (2016), Torres (2001), Liñan y Chen (2009) y Brito et al (2014) donde se plantea que si bien el espíritu emprendedor puede fomentarse como resultado del proceso de aprendizaje, la importancia que le da el joven 
emprendedor universitario a las opiniones de su entorno más cercano es lo que incide en su decisión de iniciar una empresa.

El ecosistema emprendedor universitario explica también a la intención emprendedora, aunque no tan fuertemente como la norma subjetiva, sin embargo, su rol se vuelve importante cuando se le introduce como mediador entre la norma subjetiva y la intención emprendedora. Criterio similar al realizado por Espíritu (2011) y Caicedo et al. (2015), quienes señalan a la universidad como proveedora de herramientas, habilidades y competencias que se ponen a disposición del emprendedor en su decisión de iniciar una empresa.

Por las razones anteriormente mencionadas se determina que la aprobación social por parte de la familia, amigos y profesores, para que un universitario emprenda, es fundamental y requiere de las condiciones de un ecosistema universitario apropiadas que motive y refuerce la intención a realizar emprendimientos desde una perspectiva social.

De esta manera el modelo planteado se muestra como el apropiado para explicar la intención apropiada con el constructo norma subjetiva y sus 3 sub-dimensiones como variables independientes y el ecosistema emprendedor universitario como variable mediadora.

Los resultados del presente estudio contribuyen al conocimiento académico, al menos por dos razones:

En primer lugar, con respecto al diseño de un programa educativo adecuado, que permita un aprendizaje efectivo en el que se dote al estudiante de habilidades y competencias para emprender. En segundo lugar, con respecto al panorama institucional de la formación emprendedora, donde los jóvenes se desenvuelven; en la que se promuevan mentalidades y actitudes emprendedoras y se refuerce la percepción del emprendedor en relación a su entorno familiar, social y cultural.

Una de las limitaciones del estudio es la población con la que se trabajó: estudiantes de la Facultad de Ciencias Económicas y Administrativas de la Universidad de Cuenca; la homogeneidad de la misma en algunas de las características como la edad o la dedicación, limita su capacidad predictiva y puede no ser representativa de la población general de estudiantes universitarios. Otra limitación que se podría resaltar es la observación de las variables en un momento determinado, sin realizar un seguimiento de su evolución a lo largo del tiempo.

En base a las limitaciones planteadas en el párrafo anterior, se pueden ampliar las líneas de investigación hacia estudios que profundicen el análisis de las diferentes orientaciones emprendedoras de estudiantes de las diferentes carreras profesionales, e inclusive líderes que no han accedido a estudios formales universitarios.

También se propone, considerando que la motivación emprendedora es una tarea compleja y permanente cuyos frutos se verán a largo plazo; realizar un seguimiento respecto a la evolución de las variables a lo largo del tiempo.

\section{Referencias bibliográficas}

Toledano, N., \& Urbano, D. (2007). Políticas de apoyo a la creación de empresas en España. Un estudio de casos. Boletín ICE económico, (2905), 33-46.

Kantis, H. (2010). Aportes para el diseño de políticas integrales de desarrollo emprendedor en América Latina. Desarrollo Emprendedor: América Latina y la experiencia internacional. Washington: Banco Interamericano de Desarrollo.

Díaz-García, C., Sáez-Martínez, F., \& Jiménez-Moreno, J. (2015). "Evaluación del impacto del programa educativo "Emprendedores" en la intención emprendedora de los participantes" [artículo en línea]. RUSC. Universities and Knowledge Society Journal. Número 12. UOC. Recuperado de: http://rusc.uoc.edu/rusc/es/index.php/rusc/ar ticle/view/v12n3-diaz-saez-jimenez/2643.html

SELA (2016). Experiencias recientes sobre programas de emprendimiento e incubadoras de empresas en América latina y el Caribe: Hacia la creación de una economía impulsada por el emprendimiento. Presentado en el seminario - 
Taller sobre Emprendimiento en Bridgetown, Barbados. Recuperado de http://www.sela.org/media/2303968/ experiencias-recientes-programas-de-emprendimiento.pdf

Urbano, D., Urbano, D., Díaz, C., Hernández, R., Rialp, A., Rialp, J., ... \& Aponte, M. (2006). Factores condicionantes de la creación de empresas en Cataluña: Un enfoque institucional. Estudios de Economía Aplicada, 24(2), 24-37.

OCDE (2013), Startup América Latina: promoviendo la innovación en la región, Estudios del Centro de Desarrollo, OECD Publishing.

Barrado, B., \& Molina, J. A. (2015). Factores macroeconómicos que estimulan el emprendimiento. Un análisis para los paises desarrollados y no desarrollados. Documento de Trabajo, 06.

SELA (2016). Experiencias recientes sobre programas de emprendimiento e incubadoras de empresas en América latina y el Caribe: Hacia la creación de una economía impulsada por el emprendimiento. Presentado en el seminario Taller sobre Emprendimiento en Bridgetown, Barbados. Recuperado de http://www.sela.org/media/2303968/ experiencias-recientes-programas-de-emprendimiento.pdf

Espíritu Olmos, R. (2011). Análisis de la intención emprendedora en estudiantes universitarios a través de los rasgos de personalidad. Multiciencias, Enero-Abril, 65-75. Recuperado de: http://www.redalyc.org/articulo.oa?id=90 418851009

Formichella, M. (2004). El concepto de emprendimiento y su relación con la educación, el empleo y el desarrollo local. Buenos Aires, Argentina. Recuperado de: http://municipios.unq.edu.ar/modules/mislibros/archivos/Monogr afiaVersionFinal.pdf

Samaniego Erazo, F. (2014). "Análisis y perspectiva del emprendimiento empresarial ecuatoriano en el contexto de la política económica del buen vivir", en Observatorio de la Economía Latinoamericana, Número 201. Recuperado de: http://www.eumed.net/cursecon/ecolat/ec/2014/emprendedores.html.

RIMISP, Centro Latinoamericano para el Desarrollo Rural, (2018); Pobreza y desigualdad informe latinoamericano 2017; Chile.

Instituto Nacional de Estadísticas y Censos, INEC (2017). Panorama Laboral y Empresarial del Ecuador 2017. Ecuador.

Genescá Garrigosa, E., \& Veciana Verges, J. M. (1984). Actitudes hacia la creación de empresas. Información comercial española, (611), 147-155.

Jiao, H. (2011). A conceptual model for social entrepreneurship directed toward social impact on society. Social Enterprise Journal, 7(2), 130-149.

Garzozi, R., Messina, M., Moncada, C., Ochoa, J., Ilabel, G., \& Zambrano, R. (2014). Planes de negocios para emprendedores. Iniciativa Latinamericana de libros de texto Abiertos. LATIn. Primera Edición. Recuperado de: http://www.proyectolatin.org/books/Plan_de_Negocios_para_Emprendedores_CC_BY-SA_3.0.pdf

Jennings, (1994). Multiple perspectives of entrepreneurship.

Dees, J. G. (1998). Enterprising nonprofits. Harvard business review, 76, 54-69.

Schumpeter, J. (1942). Creative destruction. Capitalism, socialism and democracy, 825, 82-85.

Drucker, P. F. (1982). El empresario de la nueva era: Diálogos en Pomona.

Hurtado, J. A. (2016). Análisis de las fallas de implementación de la politica pública: el caso de la política de fomento y apoyo al emprendimiento en Ecuador (2009-2014) (Master's thesis, Quito, Ecuador: Flacso Ecuador).

Lederman, D., Messina, J., Pienknagura, S., \& Rigolini, J. (2014). El emprendimiento en América Latina: muchas empresas y poca innovación. World Bank Publications.

Alcaraz, Rafaél. (2011); El Emprendedor de éxito, Cuarta Edición, Mc Graw Hill, México. Recuperado de: http://ww w.academia.edu/16526264/El_Emprendedor_de_\%C3\%89xito._Rafael_Alcaraz_4_edici\%C3\%B3n

Drucker, P. F. (1985). The discipline of innovation. Harvard business review, 63(3), 67-72.

Lopez de Toro, C. (2014) Caracteristicas de emprendimiento social de los jóvenes en estudios previos a los universitarios. (Trabajo fin de Máster). Recuperado de http://eprints.ucm.es/27578/

Rodríguez Lozano, Z. P., \& Larrota Vásquez, D. A. (2013). Creación de empresa ingeniería, consultoría y construcción de Colombia Ingecolco Soluciones. 
Vernis, A., \& Iglesias, M. (2010). Empresas que inspiran futuro. Ocho casos de emprendedores sociales. Barcelona: ESADE. Alvord, S. H., Brown, L. D., \& Letts, C. W. (2004). Social entrepreneurship and societal transformation: An exploratory study. The journal of applied behavioral science, 40(3), 260-282.

Zahra, S. A., Rawhouser, H. N., Bhawe, N., Neubaum, D. O., y Hayton, J. C. (2008). Globalization of social entrepreneurship opportunities. Strategic Entrepreneurship Journal.

Guzmán Vásquez, A. y Trujillo Dávila, M. A. (2008). Intraemprendimiento: Una revisión al constructo teórico, sus implicaciones y agenda de investigación futura. Cuadernos de Administración, 21(35).

Bechard, J. P., \& Toulouse, J. M. (1998). Validation of a didactic model for the analysis of training objectives in entrepreneurship. Journal of business venturing, 13(4), 317-332.

Brito, P. M., Cruz, A. B., \& Hernández, A. I. L. (2014). Un paso más en la investigación de la intención emprendedora del estudiante universitario: GUESSS. Revista de Estudios Empresariales. Segunda Época, (2). Recuperado de: h ttps://revistaselectronicas.ujaen.es/index.php/REE/article/view/1758

Shapero, A., \& Sokol, L. (1982). The social dimensions of entrepreneurship.

Ajzen, I. (1991): "The theory of planned behavior". Organizational Behavior and Human Decision Processes. Vol. 50, no 2.

Prodan, I., \& Drnovsek, M. (2010). Conceptualizing academic-entrepreneurial intentions: An empirical test. Technovation, 30(5-6), 332-347.

Harrison, G. W., \& List, J. A. (2004). Field experiments. Journal of Economic literature, 42(4), 1009-1055.

Sáez-Martínez, Francisco; Jiménez-Moreno, Juan; Díaz-García, Cristina; (2015). Evaluación del impacto del programa educativo "Emprendedores" en la intención emprendedora de los participantes. RUSC. Universities and Knowledge Society Journal, Julio-Sin mes, 17-31

Toledano Garrido, N. (2006). Las perspectivas empresariales de los estudiantes universitarios: un estudio empírico. Revista de Educación. $N^{\circ} 341$, págs. 803-825. ISSN 0034-8082. Recuperado de: http://rabida.uhu.es/dspace/bi tstream/handle/10272/10853/Las_perspectivas_empresariales.pdf?sequence $=2$

Liseras, N., de Rearte, G., María, A., \& Graña, F. M. (2003). Factores asociados a la vocación emprendedora en alumnos universitarios. Presentado en la VIII reunión anual Red Pymes Mercosur en Rosario Argentina

García, R. F., Remírez, M. P. F., Rodríguez, P. G., Martín, J. L., Nieva, M. L. J., Ripol, M. S. O., ... \& Carbó, A. R. (2006). Transformando la escuela: comunidades de aprendizaje (Vol. 36). Graó.

Díaz-Bretones, F., Tamayo, I., Parragué, M., \& Farías, A. (2011). Evaluación de Prácticas de Responsabilidad Social Corporativa: comparación de las empresas españolas y chilenas. Fundación Carolina. Proyecto CeALCI.

Pizarro, M. P. (2003). Educación, Democracia y Participación. Revista Enfoques Educacionales, 5(1), 101-105.

Echeverría, J. (2009). Participación ciudadana y educación: ¿cómo la educación puede ampliar las posibilidades de participación? Polémika, 2(1).

Torres, R. M. (2001). Participación ciudadana y educación. Una mirada amplia y 20 experiencias en América Latina. Instituto Fronensis. Quito, EC. 86p.

Soria-Barreto, K., Zuniga-Jara, S., \& Ruiz-Campo, S. (2016). Educación e Intención Emprendedora en Estudiantes Universitarios: Un Caso de Estudio. Formación universitaria, 9(1), 25-34.

Duval\#Couetil, N. (2013). Assessing the impact of entrepreneurship education programs: Challenges and approaches. Journal of Small Business Management, 51(3), 394-409.

De Luco, G. A. M. (2014). La intención emprendedora en estudiantes universitarios: el caso de la universidad de Deusto/entrepreneurial intention among university students: the case of the university of Deusto. Boletin de estudios económicos, 69(211), 151. Recuperado de: https://search.proquest.com/docview/1537380750?pq-orig site $=$ gscholar

Liñán, F., \& Chen, Y. W. (2009). Development and Cross\#Cultural application of a specific instrument to measure entrepreneurial intentions. Entrepreneurship theory and practice, 33(3), 593-617.

Ajzen, I. (2002). Perceived behavioral control, self\#efficacy, locus of control, and the theory of planned behavior 1. Journal of applied social psychology, 32(4), 665-683. 
Pedro F. Mora Pacheco, et al. Jóvenes universitarios y su apuesta al emprendimiento social

Liñán, F., \& Chen, Y. W. (2006). Testing the entrepreneurial intention model on a two-country sample.

Caicedo, G. R., Peralta, V. P., Rodríguez, A. N., \& Quintero, L. (2015). Caracterización del Ecosistema Emprendedor en las Instituciones de Educación Superior en Valledupar, Colombia. Carlos Arturo Robles Julio, 107. Recuperado de:https://www.researchgate.net/profile/Aldrin_Carvajal2/publication/308764256_USO_Y_A DICCION_A_LAS_MEDIACIONES_TECNOLOGICAS_TIC_EN_LOS_PROCESOS_PEDAGOGIC OS_CON_LOS_ESTUDIANTES_DE_LAS_FACULTADES_DE_INGENIERIA_Y_DE_EDUCACIO N_DE_LA_UNIVERSIDAD_DE_LA_GUAJIRA_cuyo_autor_es_CARVAJAL_/links/57ee94aa08ae886b 8973fdd $4 /$ USO-Y-ADICCION-A-LAS-MEDIACIONES-TECNOLOGICAS-TIC-EN-LOS-PROCESO S-PEDAGOGICOS-CON-LOS-ESTUDIANTES-DE-LAS-FACULTADES-DE-INGENIERIA-Y-DE-ED UCACION-DE-LA-UNIVERSIDAD-DE-LA-GUAJIRA-cuyo-autor-es-CARVAJAL.pdf\#page=107

Veciana, J. M., y Urbano, D. (2004). Actitudes de los estudiantes universitarios hacia la creación de empresas: un estudio empírico comparativo entre Catalunya y Puerto Rico. El emprendedor innovadory la creación de empresas de I+ D+ I, Universidad de Valencia, 35-58. Recuperado de: https://books.google.es/books?hl=es\&lr=\&id=aI WzyLD4PUEC\&oi=fnd\&pg=PA35\&dq=VECIANA+Y+URBANO\&ots=kjC1PZKt5_\&sig=RI9YnmL8 Nimrzr7ZccA6AXhhk_8\#v=onepage\&q=VECIANA\%20Y\%20URBANO\&f=false

Aponte, M., Urbano, D. y Veciana, J., (2006). Actitudes hacia la creación de empresas: Un estudio comparativo entre Catalunya y Puerto Rico. Forum Empresarial,diciembre, 52-75.

Cervantes, A. L. A., Ojeda, M. A. M., \& Martínez, R. G. (2016). Intención emprendedora en estudiantes de la Licenciatura de Administración en una Institución de Educación Superior. Presentado en el XXI Congreso Internacional de Contaduría, Administración e Informática. México

Ayaviri, A., Melo, A., y Saucedo, H. (2018). Medición de la intención de emprendimiento en universitarios mediante PLS-SEM.

Chin, W. (1998). Issues and Opinión on Structural Equation Modeling, MIS QUARTERLY, 22(1)

Cepeda, G., y Roldán, J. (2004). Aplicando en la práctica la técnica pls en la Administración de Empresas. En Conocimiento y Competitividad: Congreso ACEDE, Murcia

Barclay, D., Higgins, C., Thompson, R. (1995). The partial Least Squares (PLS) Aproach to Causal Modelling: Personal Computer Adoption an Use as an Illustration, Technology Studies, Special Issue on Research Methodology

Chin, W., Marcolin, B., \& Newsted, P. (2003). Apartial least squares latent variable modeling approach for measuring interaction effects: results from a Monte Carlo simulation study and electronic, mail emotion / adoption study. Information System Research.

Universidad San Martín de Porres USMP (2017) Lima - Perú, proyecto "Potencial para el Emprendimiento Social en América Latina".

Moriano, J. A. (2005). El perfil psicosocial del emprendedor [The psychosocial profile of the entrepreneur]. Madrid: Consejo Económico y Social.

Giraldo W. \& Vara-Horna A. (2018). "El Emprendimiento Social de Base Universitaria en Latinoamérica. ¿Están las Universidades haciendo lo suficiente?” Lima - Perú.

Sánchez Almagro, M. L., Moriano León, J. A., \& Sevilla Solano, C. (2003). Guía Luces para emprendedores. Patronato Pedro de Ibarra. Cáceres, España, 286.

Franke, N., \& Lüthje, C. (2004). Entrepreneurial intentions of business students-A benchmarking study. International Journal of Innovation and Technology Management, 1(03), 269-288.

Alvarez-Risco, A., López-Odar, D., \& Chafloque-Céspedes, R. (2018). Conducta Ambiental en Estudiantes de Perú.

Astudillo, S., Mora, P. y Pozo, S. (2019). Evaluación de la cátedra de emprendimiento desde su intención emprendedora en una universidad pública en Cuenca (Ecuador). Brazilian Journal of Development, 5(5), 3770-3785. Recuperado de: http://brjd.com.br/index.php/BRJD/article/view/1546/1701

Bilbao, N. S., y Vélez, A. L. L. (2015). Las competencias de emprendimiento social, COEMS: Aproximación a través de programas de formación universitaria en Iberoamérica. REVESCO. Revista de Estudios Cooperativos, (119), 159-182. 
Giraldo-Mejía, W. A., \& Vara-Horna, A. (2018). UNIVERSITY-BASED SOCIAL ENTREPRENEURSHIP IN LATIN AMERICA / EL EMPRENDIMIENTO SOCIAL DE BASE UNIVERSITARIA EN LATINOAMÉRICA. Universidad San Martín de Porres, Lima. Perú. Recuperado de: https://www.researchgate.net/profile/Dennis_Odar/ publication/329388662_UNIVERSITY-

BASED_SOCIAL_ENTREPRENEURSHIP_IN_LATIN_AMERICA_Are_universities_doing_enough/ links/5c05fd9aa6fdcc315f9ae923/UNIVERSITY-BASED-SOCIAL-ENTREPRENEURSHIP-IN-LATINAMERICA-Are-universities-doing-enough.pdf

\section{Notas}

[1] Economista por la Universidad de Cuenca, Ecuador. Magister en Gerencia y Liderazgo Educacional. Docente titular e investigador de la Facultad de Ciencias Económicas y Administrativas de la Universidad de Cuenca, Ecuador. ORCID: 0000-0002-5220-5118

[2] Ingeniero Financiero por la Universidad de Cuenca, Ecuador. Abogado por la Universidad Técnica Particular de Loja, Ecuador, Magister en Auditoría Integral. Master Universitario en Dirección Financiera y Contable de la Empresa. Docente investigador de Facultad de Ciencias Económicas y Administrativas de la Universidad de Cuenca, Ecuador. ORCID: 0000-0002-8953-8151

[3] Ingeniera Comercial por la Universidad de Cuenca, Ecuador. Magister en Gerencia y Liderazgo Educacional. Docente titular e investigador de la Facultad de Ciencias Económicas y Administrativas de la Universidad de Cuenca, Ecuador. ORCID: 0000-0002-4382-4689

[4] Ingeniero Comercial por la Universidad del Azuay, Ecuador. Doctor en Jurisprudencia por la Universidad del Azuay, Ecuador. Master of Science Entrepreneurship por la Oklahoma State Univerity, Estados Unidos. Docente titular e investigador de la Facultad de Ciencias Económicas y Administrativas de la Universidad de Cuenca, Ecuador. ORCID: 0000-0003-0044-4333

(C) Universidad de Cuenca 2019

CC BY-NC-SA

INFORMACIÓN ADICIONAL

Código JEL:: M13 